\title{
The nature of nitrogen related point defects in common forms of InN
}

\author{
K. S. A. Butcher, ${ }^{\text {a) }}$ A. J. Fernandes, P. P.-T. Chen, and M. Wintrebert-Fouquet \\ Physics Department, Macquarie University, Sydney, New South Wales 2109, Australia \\ H. Timmers and S. K. Shrestha \\ School of Physical, Environmental, and Mathematical Sciences, University of New South Wales \\ at the Australian Defence Force Academy, Canberra ACT 2600, Australia \\ H. Hirshy and R. M. Perks \\ Cardiff School of Engineering, Cardiff University, The Parade, Cardiff CF24 3AA, United Kingdom \\ Brian F. Usher \\ Department of Electronic Engineering, La Trobe University, Bundoora VIC 3086, Australia
}

(Received 11 September 2006; accepted 30 March 2007; published online 18 June 2007)

\begin{abstract}
The role of point defects related to the presence of excess nitrogen is elucidated for $\mathrm{InN}$ thin films grown by different techniques. Elastic recoil detection analysis has shown the presence of excess nitrogen in state-of-the-art InN films. Using $\mathrm{x}$-ray photoelectron spectroscopy and $\mathrm{x}$-ray diffraction it is shown that two distinct forms of point defects can be distinguished; one of these appears to be an interstitial form of nitrogen, common in some forms of polycrystalline InN. The other is associated with a combined biaxial and hydrostatic strain observed for molecular beam epitaxy (MBE) and chemical vapor deposition (CVD) grown films, and may be a mixture of the nitrogen-on-metal antisite defect and lower densities of indium vacancies and interstitial nitrogen. The high density of defects present in all the InN samples examined suggests that stoichiometry related point defects dominate the electrical and optical properties of the material. The difference in the type of point defect observed for polycrystalline ( $\mathrm{rf}$ sputtered) and epitaxial (MBE and CVD) InN supports existing evidence that the Moss-Burstein effect is not an adequate description of the apparent band-gap difference between InN samples grown by different techniques. (C) 2007 American Institute of Physics. [DOI: 10.1063/1.2736654]
\end{abstract}

\section{INTRODUCTION}

The volatility of nitrogen led to an early expectation that all InN films would be metal rich; however, several techniques have now shown that many state-of-the-art InN films tend to contain excess nitrogen. Using elastic recoil detection analysis (ERDA) up to 33\% excess nitrogen has been reported for some forms of InN. ${ }^{1-3}$ Energy dispersive X-ray (EDX) analysis has confirmed such results. In addition UVRaman measurements have indicated a "near-surface" molecular species present when high excess nitrogen is observed. ${ }^{2}$ In this paper we present further data, collected using high resolution $\mathrm{x}$-ray photoelectron spectroscopy (XPS), which unambiguously confirms the presence of nitrogen related defects in InN films grown by a number of techniques. We also compare $\mathrm{x}$-ray diffraction (XRD) and XPS data with ERDA results to provide some insight into the possible origin of point defects that might be related to nitrogen rich $\mathrm{InN}$ stoichiometry.

Improvements in the crystallinity and electronic properties of molecular beam expitaxy (MBE) grown InN over the last five years have resulted in a marked increase in interest in this material. ${ }^{5,6}$ However, despite this improvement in material quality, compared to other semiconducting materials, $\mathrm{InN}$ is still limited to extremely high carrier concentrations,

\footnotetext{
a) Author to whom correspondence should be addressed. Tel.: 61 (0)29850 8916; Fax: 61 (0)29850 8115; electronic mail: sbutcher@ics.mq.edu.au
}

in excess of $1 \times 10^{17} \mathrm{~cm}^{-3}$, while the maximum electron mobility of $2100 \mathrm{~cm}^{2} / \mathrm{V}$ s so far achieved for MBE grown material $^{7}$ is only about half the value of the theoretical maximum. ${ }^{8,9}$ The defect structure of $\mathrm{InN}$ is therefore a particularly important topic. At present the material physics of $\mathrm{InN}$ is only partially understood. Some original assumptions have had to be revised. For instance, the assertion that the electron carrier concentration dependence of the optical absorption edge for epitaxial material was due entirely to a Moss-Burstein effect has been shown to be incorrect. It has been demonstrated that this phenomenon accounts for less than $60 \%$ of the apparent change in the band gap represented by the curve in Fig. $1 .^{10}$ Bulk oxygen and other impurity species, with the possible exception of hydrogen, appear to be at concentrations too low to account for the carrier concentrations observed. ${ }^{11-13}$ As shown in this paper, the deviations from 1:1 nitrogen to indium stoichiometry represent the inclusion of native defects at far higher densities than the combined foreign impurity content. Native defects are therefore a distinct possibility as a source of the high background carrier concentration of $\mathrm{InN}$, and even in the presence of hydrogen considerable film nonstoichiometry would result if the hydrogen was not interstitial. At the highest carrier concentrations observed for InN, percentage amounts of carriers would have to be present to act as donors. Stoichiometry variations can account for such high levels. Native defects present at these high concentrations will have an impact on both the optical absorption properties and on the crystal lat- 


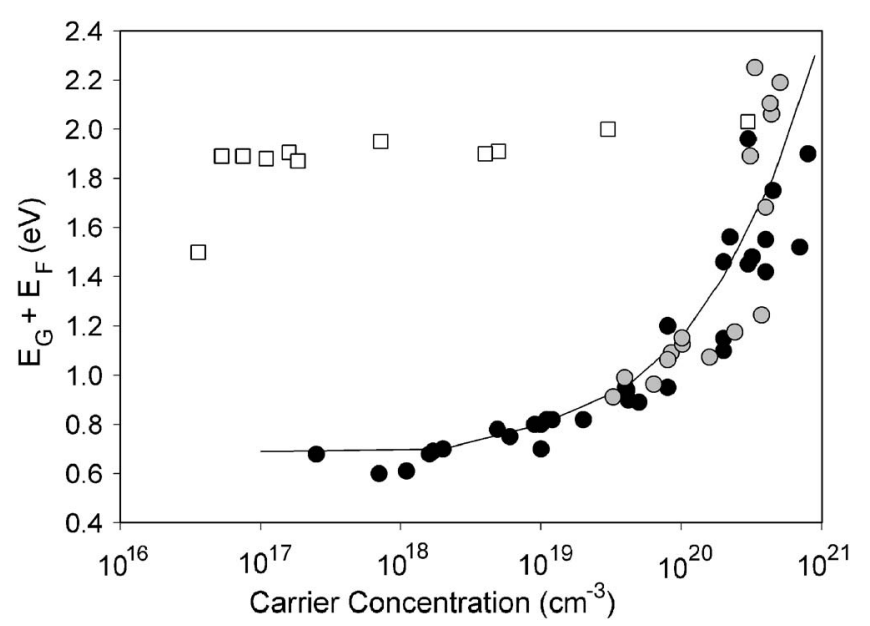

FIG. 1. Band-gap energy plus Fermi-level energy (from absorption square plots) vs carrier concentration for a selection of MBE (black circles), RPECVD (gray circles), and rf sputtered (white squares) data taken from Ref. 6.

tice constants. Large changes in the regular lattice arrangement will also affect the value of the band gap itself. Hence, the Moss-Burstein effect cannot be the only phenomenon that influences the optical absorption edge of $\mathrm{InN}$.

Nonetheless, it is interesting that a substantial number of epitaxial InN samples have been reported to follow the same carrier concentration versus absorption edge trend represented by the curve in Fig. 1 (though with some notable exceptions ${ }^{6,10}$ ), and although the Moss-Burstein effect does not completely explain this trend, it would appear that a common defect structure might be present for such samples. Figure 1 displays the optical absorption edge (from absorption square measurements) versus carrier concentration data for a selection of samples taken from Ref. 6. For this paper we have studied epitaxial films at the higher absorption-edge part of the epitaxial material trend, given by the curve in Fig. 1. As shown elsewhere, ${ }^{6,10}$ and as indicated in Fig. 1, other forms of InN do not follow the trend shown in the figure and are in clear violation of the proposed Moss-Burstein solution of apparent band-gap variation. These films appear to have consistently high apparent band gaps as measured by optical absorption. We have also studied the defect structure of some of these materials.

Assumptions regarding the propensity of $\mathrm{InN}$ to be nitrogen deficient are also challenged here. It has long been assumed that nitrogen vacancies were the dominant native defect for InN. ${ }^{5,6}$ Ho et al. ${ }^{14}$ have recently reported carrier concentrations of $4 \times 10^{21} \mathrm{~cm}^{-3}$ for MBE InN purposely grown using indium rich conditions-supporting the case for nitrogen vacancies as a possible donor species for InN. However, high carrier concentrations have also been observed for extremely nitrogen rich $\mathrm{InN},{ }^{2,12,15}$ suggesting that for film growth under certain conditions other native defects may be important. In the following sections we establish the veracity of the measurements for the nitrogen rich material and then attempt to determine the possible native defect structure of such material.

\section{FILM PROPERTIES}

As outlined above, the samples studied for this paper represent two broad groups of $\mathrm{InN}$ materials: epitaxial samples, which appear to approximately follow the upper and middle sections of the defect line represented in the plot of absorption edge versus carrier concentration given in Fig. 1 , and rf sputtered polycrystalline samples that do not follow this trend, but are also represented in Fig. 1. The defect line shown in the figure was previously thought to represent a severe Moss-Burstein effect for epitaxial InN samples, whereby a $\sim 0.65 \mathrm{eV}$ band gap was modified to a higher absorption edge by the presence of a high electron carrier concentration in the conduction band. It has since been shown that the Moss-Burstein effect can only account for a maximum of $60 \%$ of the change in absorption edge: for a material with a $5 \times 10^{20} \mathrm{~cm}^{-3}$ electron carrier concentration. The maximum extent of conduction band filling with free electrons can be delineated using temperature dependent absorption measurements since tailing states at the bottom of the conduction band have a different energy dependence than the free electrons present in the conduction band itself. On this basis it was shown that the maximum extent of the Moss-Burstein effect at this carrier concentration was $0.72 \mathrm{eV}$ and that the resulting band gap for a sample with absorption edge at $1.88 \mathrm{eV}$ had to be at or above $1.16 \mathrm{eV} .^{10}$ This value is quite different to the $\sim 0.65 \mathrm{eV}$ band gap claimed for a lower carrier concentration epitaxial material. Despite this difference and the failure of the Moss-Burstein effect to explain all of the observed variations in absorption edge, it appears that samples that approximately follow the defect line of Fig. 1 may be dominated by a common set of defects.

In contrast, the nonepitaxial polycrystalline material studied here appears to have some very different optical properties compared to the epitaxial material, as is shown in Fig. 1, and is often referred to as the "high band-gap material."

InN thin films produced by four different techniques were examined during this work. MBE materials were supplied by Cornell University and the Ioffe Institute. The Ioffe MBE material was grown epitaxially directly on sapphire substrates. The Cornell material was grown epitaxially on a thick $200 \mathrm{~nm}$ AlN template as described elsewhere, ${ }^{16}$ and this material has been shown to have oxygen levels sometimes as low as $3 \times 10^{16} \mathrm{~cm}^{-3}$, while other bulk impurity levels, with the possible exception of hydrogen, have also been shown to be at too low a concentration level to explain the bulk carrier concentration. ${ }^{11}$ A number of native defects have been suggested as possible donors in $\mathrm{InN} ;{ }^{6,17}$ therefore, in the presence of the relatively large values of nonstoichiometry the influence of native defects needs to be seriously considered.

Remote plasma enhanced chemical vapor deposition (RPE-CVD), rf sputtered and reactively evaporated $\mathrm{InN}$ thin films grown at Macquarie University were also examined. The RPE-CVD material was grown epitaxially on sapphire and was also grown on glass substrates. This method of growth is described in a number of related publications. ${ }^{12,15,18}$ The bulk oxygen content of the RPECVD samples has been measured by secondary ion mass spectroscopy (SIMS) and found to generally be much less than the film carrier concentrations (sometimes by up to two 
orders of magnitude). ${ }^{12}$ The rf sputtered material was polycrystalline, with $c$-axis oriented grains, and was grown on glass or silicon substrates. The rf sputtered samples examined here had high carrier concentrations above $10^{20} \mathrm{~cm}^{-3}$. The oxygen content of this material generally varied between $3 \%-20 \%$ dependent on the size of the grains in the film. As discussed below in Sec. V and elsewhere, ${ }^{5,6}$ it has been proposed that the oxygen in these films forms on exposure of the films to the ambient atmosphere so that an amorphous layer of hydroxide and oxide species is present at the grain boundaries. The internal bulk of the crystals formed by rf sputtering has relatively little oxygen present, and no crystalline indium oxide is observed by x-ray diffraction, which would otherwise indicate the incorporation of oxygen during the actual film growth.

Like the rf sputtered material, the thin films grown by reactive evaporation were polycrystalline and were grown on glass or sapphire substrates. The reactively evaporated material has a similar oxygen content as the rf sputtered $\mathrm{InN}$, but is different in that the carrier concentrations are reproducibly low in comparison to the rf sputtered material, with values typically in the range of $2 \times 10^{17}-5 \times 10^{19} \mathrm{~cm}^{-3}$. Absorption measurements in the visible to near infrared range confirm the low carrier concentrations of this material since the strong free electron absorption visible for $\mathrm{InN}$ samples with higher carrier concentrations is generally absent, as shown in Fig. 2. The variable apparent band gap observed in the absorption measurements of the figure does not appear to show any significant carrier concentration dependence. Preliminary results, discussed elsewhere (as are details of the film growth for the reactively evaporated samples) ${ }^{10}$ indicate that the variation is, however, related to material stoichiometry.

Though for this study, as described in the following sections, a number of measurement techniques were applied to most of the samples, for the reactively evaporated samples only XPS measurements were carried out, and these measurements were only performed for comparison with the XPS data of the higher carrier concentration rf sputtered material.

\section{ELASTIC RECOIL DETECTION ANALYSIS RESULTS}

In this section we present a selection of our ERDA measurements on InN samples for comparison with the XRD results. Large variations from ideal 1:1 stoichiometry, when present, can be easily observed for many materials using a number of techniques. However, typical methods for determining metal-nitride ratios for nitride compounds, although in some cases having a good precision, lack the accuracy required to help understand structural and electronic properties. Rutherford backscattering spectroscopy (RBS), under best experimental conditions, is $3 \%-5 \%$ accurate for determining elemental concentrations. ${ }^{19,20}$ Furthermore, the nitrogen signal in RBS is typically obscured by the signal from the substrate elements so that even poorer accuracy is achieved. Energy and wavelength dispersive spectroscopies, which require calibration standards, have a stoichiometric accuracy of approximately 3\%-5\%. Secondary ion mass spectroscopy, also requiring standards, can only be regarded
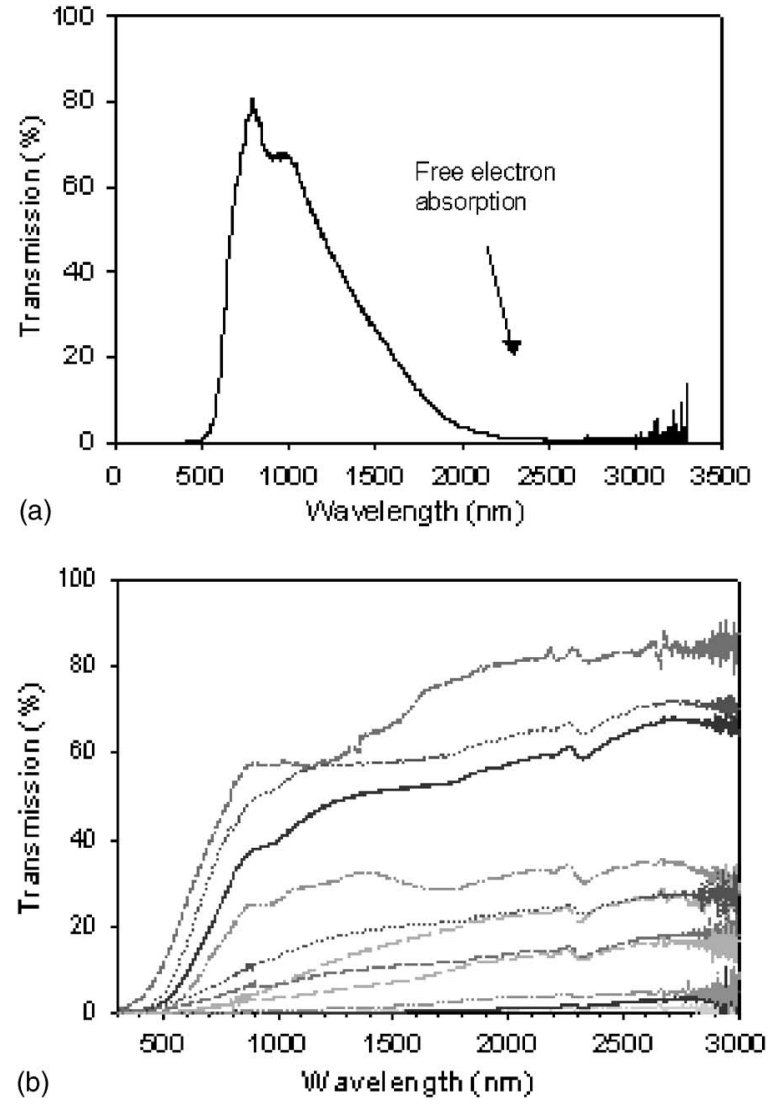

FIG. 2. (a) Absorption data for a rf sputtered InN sample with carrier concentration over $10^{20} \mathrm{~cm}^{-3}$. The strong infrared absorption is free electron absorption, which results from having a large density of electrons present in the conduction band. (b) Absorption data for a number of InN samples grown by reactive evaporation. These samples show no evidence of free electron absorption in the region measured.

as a semiquantitative technique, especially when analyzing percentage amounts of a material since matrix effects can be particularly strong. ${ }^{20} \mathrm{X}$-ray photoelectron spectroscopy and Auger electron spectroscopy are both only 10\% accurate even when using standards. ${ }^{20}$ In contrast, ERDA, which has recently been developed for the analysis of InN films, ${ }^{3,21}$ can determine the N/In ratio of a film with an accuracy of better than $2 \%$ since, in contrast to RBS, the technique intrinsically separates the nitrogen signal from the signal from other elements. In our measurements ${ }^{21}$ a $200 \mathrm{MeV} \mathrm{Au}$ ion projectile beam was incident on the $\mathrm{InN}$ samples, and recoil ions were detected at a recoil angle of $45^{\circ}$ using a position sensitive ionization detector. The detector is capable of determining both the recoil ion energy and the energy loss of the ions as they transit sections of the detector. Using heavy Au projectile ions ensures that all elements within the InN films are separated kinematically for film thicknesses up to approximately $1 \mu \mathrm{m}$. Some nitrogen loss occurs during the measurement. However, this has been demonstrated to follow the bulk molecular depletion model, and can be corrected. ${ }^{21}$ Using a sulfur projectile beam as an alternative to $\mathrm{Au}$, nitrogen loss can be avoided, and such analysis on several InN films has confirmed the results with the Au projectiles. ${ }^{22}$

Our ERDA measurements have shown that many stateof-the-art InN films have a nitrogen excess. Significantly, thin $(<1 \mu \mathrm{m})$, moderately $n$-type $\mathrm{InN}$ films grown by MBE 
TABLE I. N/In ratios measured by ERD for various rf sputtered, RPE-CVD, and MBE grown InN samples. In this case the Cornell MBE samples were grown on AlN buffer layers $200 \mathrm{~nm}$ thick; the Ioffe MBE samples were grown directly on sapphire with no intentional buffer layer. Also shown in the table is the $c$ axis lattice parameter carrier concentrations and thicknesses of the samples.

\begin{tabular}{lcccc}
\hline \hline & $\begin{array}{c}\text { Carrier } \\
\text { concentration } \\
\text { InN Sample ID }\end{array}$ & N/In & $\begin{array}{c}c \text { axis lattice } \\
\text { parameter } \\
(\AA)\end{array}$ & $\begin{array}{c}\text { Sample } \\
\text { thickness }(\mu \mathrm{m})\end{array}$ \\
\hline rf InN (20/Mar/03) & $4.7 \times 10^{20}$ & $1.21 \pm 0.03$ & 5.744 & 0.51 \\
RPE-CVD (17/May/04) & $3.1 \times 10^{20}$ & $1.34 \pm 0.01$ & 5.734 & 0.44 \\
RPE-CVD (3/Oct/03) & $4.0 \times 10^{20}$ & $1.26 \pm 0.05$ & 5.738 & 0.22 \\
RPE-CVD (15/Sep/03) & $2.5 \times 10^{20}$ & $1.15 \pm 0.05$ & 5.714 & 0.18 \\
RPE-CVD (31/Jul/03) & $3.1 \times 10^{20}$ & $1.10 \pm 0.03$ & 5.708 & 0.18 \\
RPE-CVD (19/May/04) & $1.0 \times 10^{20}$ & $1.01 \pm 0.01$ & 5.707 & 0.59 \\
RPE-CVD (22/May/03) & $8.0 \times 10^{19}$ & $1.05 \pm 0.02$ & 5.698 & 0.76 \\
RPE-CVD (26/May/04) & $8.0 \times 10^{19}$ & $1.03 \pm 0.01$ & 5.698 & 0.72 \\
MBE Ioffe InN W275 & $2.5 \times 10^{19}$ & $1.04 \pm 0.02$ & 5.7005 & 0.70 \\
MBE Ioffe InN W431 & $\cdots$ & $1.07 \pm 0.03$ & 5.7213 & 0.33 \\
MBE Cornell InN GS-1322 & $3.5 \times 10^{18}$ & $1.02 \pm 0.02$ & $\cdots$ & 0.55 \\
MBE Cornell InN GS-1337 & $5.0 \times 10^{18}$ & $1.04 \pm 0.03$ & $\ldots$ & 0.60 \\
MBE Cornell InN GS-1353 & $3.4 \times 10^{18}$ & $1.06 \pm 0.03$ & $\ldots$ & 0.50 \\
\hline \hline
\end{tabular}

tend to have a few at. $\%$ excess nitrogen present, ${ }^{3,21}$ with examples shown in Table I. Also shown in the table are the $\mathrm{N} / \mathrm{In}$ ratios for a rf sputtered sample, a material which we have previously shown to typically have very high $\mathrm{N} / \mathrm{In}$ ratios, ${ }^{1-3}$ and for InN samples grown by RPE-CVD. For the RPE-CVD material it has been shown that film growth at lower temperatures $\left(<400{ }^{\circ} \mathrm{C}\right)$ results in the incorporation of increasing amounts of excess nitrogen and that those epitaxial samples with greater excess nitrogen have a higher carrier concentration. $^{12}$

Theoretical calculations by Stampfl et al. ${ }^{17}$ show that the formation energies for excess nitrogen species in $\mathrm{InN}$ are very high. However, those calculations assume a condition of thermodynamic equilibrium during growth, and growth techniques, such as sputtering, RPE-CVD, and some types of MBE, utilize plasma sources that operate well away from conditions of thermodynamic equilibrium. The lowest potential energy for the excited reactive species created by a nitrogen plasma is greater than $6 \mathrm{eV}$. Hence, more than enough excess energy is available to establish defects with high formation energies. Defects involving excess nitrogen are therefore possible in InN when using plasma sources for film growth. Nitrogenation of substrates prior to growth is another example of a nonequilibrium treatment in MBE systems that may have some similarity to the conditions described here.

\section{HIGH RESOLUTION X-RAY PHOTOELECTRON SPECTROSCOPY RESULTS}

XPS is a mature, established technique for determining the chemical composition of surfaces. Several well regarded texts describe the technique, for example, Briggs and Seah ${ }^{23}$ and Barr. ${ }^{24}$ The latest generation of XPS systems have seen considerable improvement compared to the systems from just a few years back - the energy resolution of elemental spectra is less than half of what it was five years ago. Past XPS studies of InN have therefore shown a broad N $1 s$ pho- toelectron peak related to InN. For instance, the $\mathrm{N} 1 s \mathrm{InN}$ peak presented by Kumar et al. ${ }^{25}$ shows a full width at half maximum (FWHM) of approximately $3.75 \mathrm{eV}$. For the measurements shown here the FWHM is typically less than $1 \mathrm{eV}$. An ESCALAB 220i-XL high-sensitivity, high resolution XPS system manufactured by VG Scientific was used for the measurements. A set of typical N $1 s$ core level surface spectra is shown in Fig. 3 for representative InN films grown by MBE, RPE-CVD, rf sputtering, and reactive evaporation. All the surface spectra shown were calibrated to the $\mathrm{C} 1 \mathrm{~s}$ binding energy for adventitious carbon, known to be present at $284.8 \mathrm{eV}^{26,27}$

The $\mathrm{N} 1 s$ core level photoelectron peaks are of interest here since any peaks not related to $\mathrm{InN}$ bonding indicate a nitrogen rich defect structure. A number of $\mathrm{N} 1 s$ peaks are visible in the spectra shown in Fig. 3. Oxygen is present at the surface of all the samples studied but is most commonly bound as indium hydroxide or indium oxide and is not of interest here. Peak identifications and positions of the $\mathrm{N} 1 s$ photoelectron peaks are provided in Table II. The peaks were deconvoluted using the standard VG provided vendor software. In the past the peaks observed near $\sim 403.7 \mathrm{eV}$ have been attributed to a number of $\mathrm{N}-\mathrm{O}$ species and/or to molecular nitrogen (see the discussion in Ref. 26). Molecular nitrogen is a possibility here (though we do not discount the presence of some proportion of $\mathrm{N}-\mathrm{O}$ surface bound species) as we have previously identified a surface contribution of this species using UV Raman. ${ }^{2}$ The peak at $\sim 399.5 \mathrm{eV}$ may be attributed to $\mathrm{N}-\mathrm{H}$ species, as observed during the $\mathrm{NH}_{3}$ dosing of metals. ${ }^{28} \mathrm{~N}-\mathrm{H}$ species may be expected as a hydrolysis product of $\mathrm{InN}$ following exposure to the water in air, via the reaction

$$
\mathrm{InN}+3 \mathrm{H}_{2} \mathrm{O} \rightarrow \mathrm{NH}_{3}+\mathrm{In}(\mathrm{OH})_{3} .
$$

Partial hydrolysis of InN would, of course, result in the presence of chemisorbed $\mathrm{NH}$ and $\mathrm{NH}_{2}$ species. We have previously identified the presence of the other reaction product in Eq. (1), i.e., $\operatorname{In}(\mathrm{OH})_{3}$, at the surface of $\operatorname{InN}$, though this spe- 

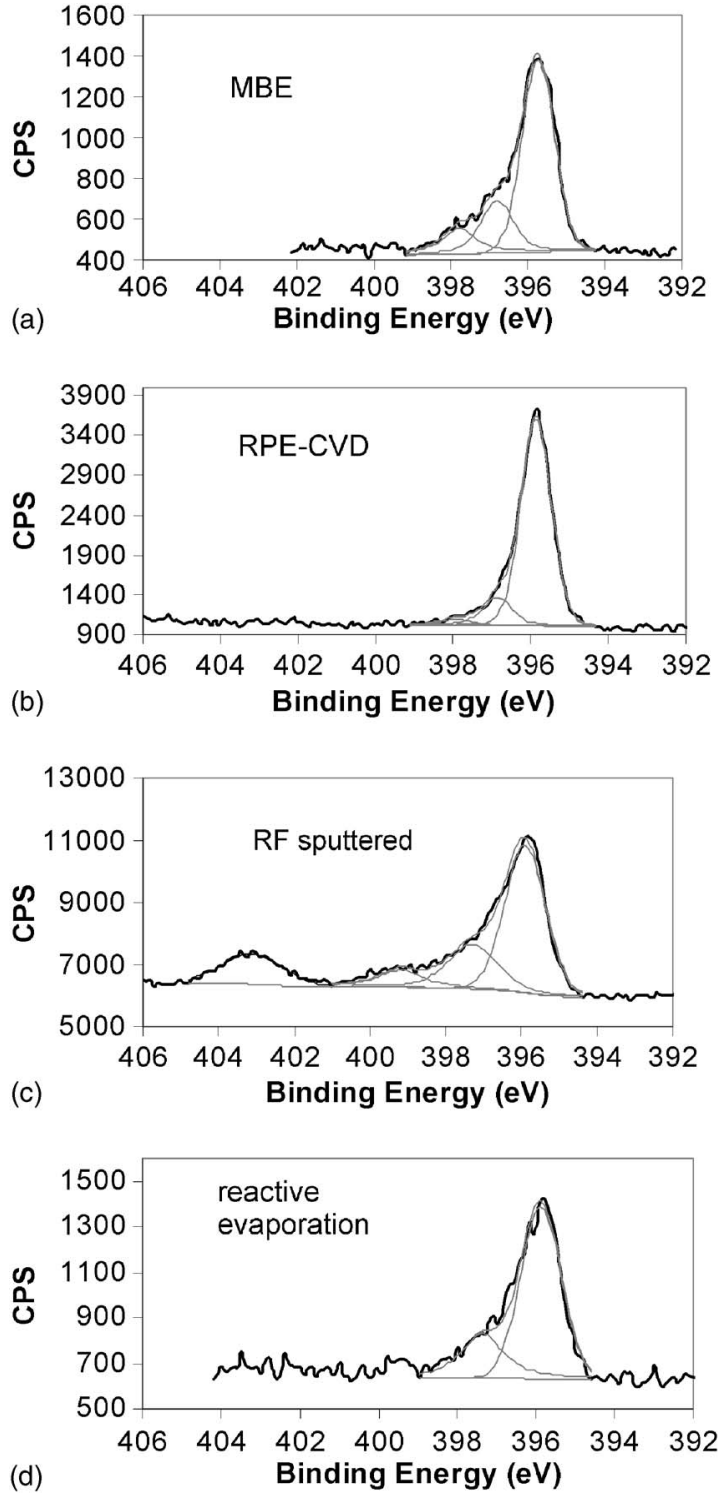

FIG. 3. The figure shows the N $1 s$ spectra obtained by XPS at the surface of indium nitride. The samples were grown by different methods. (a) MBE sample GS-1332; (b) grown by RPE-CVD on $c$-plane sapphire at $450{ }^{\circ} \mathrm{C}$, 21 May 2004; (c) grown by rf sputtering on glass, 4-5 July 2005; and (d) grown by reactive evaporation on glass, 25 January 2006. The data for (a) was measured at a different time over a slightly different energy range; however, no other nitrogen related peaks were observed in a wide energy scan.

cies is visibly converted to the oxide under the $\mathrm{Al} K \alpha$ radiation often used for XPS studies. ${ }^{29}$ In the case of one of the RPE-CVD samples the peak at $\sim 399.5 \mathrm{eV}$ may also have been due to $\mathrm{C}-\mathrm{N}$ bonding. This is discussed in further detail below.

The peak at approximately $397.8 \mathrm{eV}$ is similar in position to the $398.2 \mathrm{eV}$ peak observed by Schreifels et al. ${ }^{28}$ on dysprosium dosed with molecular nitrogen; those authors attributed the peak to chemisorbed atomic nitrogen, which apparently exists as an intermediary step in the chemical formation of a dysprosium nitride. If the species observed here at $397.8 \mathrm{eV}$ represents a form of atomic nitrogen in $\mathrm{InN}$, then its presence would explain some of the excess nitrogen observed by ERDA in the InN samples. This particular peak was not observed for RPE-CVD samples that were examined relatively soon after film growth, nor was the peak always easily observed after argon ion etching of those samples with only a small excess of nitrogen measured by ERDA. The peak was observed for all rf sputtered samples and for samples grown by reactive evaporation, regardless of their age prior to analysis. It was also present for one of the MBE samples examined, though that sample was examined some months after film growth.

The peak at $\sim 396.0 \mathrm{eV}$ has previously been attributed to $\mathrm{InN}$, albeit with lower resolution measurements, and these results are consistent with that observation. ${ }^{25}$ The smaller peak at $\sim 396.9 \mathrm{eV}$, observed only for the RPE-CVD and MBE samples, has not been observed previously because of the lower energy resolution of the XPS systems used prior to this. Of course, XPS peaks need not result from chemical shifts. A number of potential nonchemical origins were examined here. The possibility that the peak could be a shake-up peak related to the main InN N $1 s$ peak is excluded since with argon ion etching of the RPE-CVD samples this peak was observed to grow substantially in relation to the main InN N $1 s$ peak. As shown in Fig. 4, the $\sim 396.9 \mathrm{eV}$ contribution was also observed to be larger for those RPECVD samples for which ERDA showed a greater amount of excess nitrogen (i.e., for samples grown at lower temperatures), suggesting that the peak has a distinct chemical origin. The figure compares three samples with varying levels of excess nitrogen present. It is also unlikely that a shake-up peak would be present for the RPE-CVD and MBE samples, and not for the reactively evaporated or rf sputtered samples.

It may be suspected that Fermi level variations at the sample surface due to electron accumulation at the InN surface may have caused a shift of the $396.0 \mathrm{eV}$ peak to $396.9 \mathrm{eV}$. However, it can also be shown that the peak at $\sim 396.9 \mathrm{eV}$ is not related to surface variations in the Fermi level of the material. Figure 5 shows a $\mathrm{N} 1 \mathrm{~s}$ spectrum for a sample that was etched with argon ions and then heated to remove physisorbed species. The contribution at $\sim 396.9 \mathrm{eV}$ is clearly seen. The figure also shows the In $3 d$ peak for the same sample, for which there is no evidence of a similar shift in energy from the main In $3 d \mathrm{InN}$ related peak. Variations in the Fermi level with sample depth should cause the same energy shift for all the core level spectra. This is clearly not observed here.

For the polycrystalline rf sputtered and reactively evaporated samples, there also appears to be no reliance of the $\mathrm{N}$ $1 s$ spectra on Fermi level effects since, as explained in Sec. II, the reactive evaporation samples have a relatively low carrier concentration compared to the rf sputtered material; yet, as shown in Table II, there is no discernible change in the $\mathrm{N} 1 s$ peak positions related to the difference in carrier concentration of the two materials. The reactively evaporated polycrystalline material also spans the carrier concentrations of the RPE-CVD and MBE samples, which suggests that the presence of the $\sim 396.9 \mathrm{eV}$ peak in the epitaxial material, and not in the polycrystalline materials, is again unrelated to variations in the Fermi level of the materials studied.

Egelhoff, $\mathrm{Jr}^{30}$ has reviewed the mechanisms that lead to changes in binding energy for XPS core level peaks. It is 
TABLE II. N $1 s$ core photoelectron surface positions for MBE, RPE-CVD, RF sputtered, and reactively evaporated InN samples.

\begin{tabular}{|c|c|c|c|c|c|}
\hline \multirow[b]{2}{*}{ Sample } & \multicolumn{5}{|c|}{ Core level assignment $(\mathrm{eV})$} \\
\hline & InN & Defect & Atomic N & $\begin{array}{l}\mathrm{N}-\mathrm{H} \text { or } \\
\mathrm{C}-\mathrm{N}\end{array}$ & $\begin{array}{c}\mathrm{N}-\mathrm{O} \text { or } \\
\text { molecular } \mathrm{N}\end{array}$ \\
\hline $\begin{array}{c}\text { MBE } \\
\text { GS-1322 }\end{array}$ & 395.59 & 396.69 & $\cdots$ & $\cdots$ & $\cdots$ \\
\hline $\begin{array}{l}\text { MBE } \\
\text { W431 }\end{array}$ & 395.71 & 396.72 & 397.92 & $\cdots$ & $\cdots$ \\
\hline $\begin{array}{l}\text { RPE-CVD }(25-26 / \text { May/04) } \\
\text { at } 200{ }^{\circ} \mathrm{C} \text { on glass }\end{array}$ & 395.99 & 397.08 & 397.99 & 399.49 & $\cdots$ \\
\hline $\begin{array}{l}\text { RPE-CVD }(18 / \mathrm{May} / 04) \\
\text { at } 300{ }^{\circ} \mathrm{C} \text { on glass }\end{array}$ & 395.90 & 396.98 & 398.09 & $\cdots$ & $\cdots$ \\
\hline $\begin{array}{l}\text { RPE-CVD (18/May/04) } \\
\text { at } 300{ }^{\circ} \mathrm{C} \text { on sapphire }\end{array}$ & 395.87 & 396.89 & 397.86 & $\cdots$ & $\cdots$ \\
\hline $\begin{array}{l}\text { RPE-CVD }(19-20 / \text { May/04) } \\
\text { at } 400{ }^{\circ} \mathrm{C} \text { on glass }\end{array}$ & 395.65 & 396.74 & 397.74 & $\cdots$ & $\cdots$ \\
\hline $\begin{array}{l}\text { RPE-CVD }(21 / \text { May/04 }) \\
\text { at } 450{ }^{\circ} \mathrm{C} \text { on glass }\end{array}$ & 395.74 & 396.84 & 397.92 & $\cdots$ & $\cdots$ \\
\hline $\begin{array}{l}\text { RPE-CVD }(21 / \text { May/04) } \\
\text { at } 450{ }^{\circ} \mathrm{C} \text { on sapphire }\end{array}$ & 395.87 & 396.86 & 397.92 & $\cdots$ & $\cdots$ \\
\hline $\begin{array}{l}\text { RPE-CVD }(22-23 / \mathrm{May} / 04) \\
\text { at } 500{ }^{\circ} \mathrm{C} \text { on glass }\end{array}$ & 395.61 & 396.74 & $\cdots$ & $\cdots$ & $\cdots$ \\
\hline $\begin{array}{l}\text { RPE-CVD }(27 / \mathrm{May} / 04) \\
550{ }^{\circ} \mathrm{C} \text { on glass }\end{array}$ & 395.71 & 396.97 & $\cdots$ & $\cdots$ & $\cdots$ \\
\hline $\begin{array}{l}\text { RPE-CVD }(5 / \mathrm{Jan} / 05) \\
510^{\circ} \mathrm{C} \text { on sapphire }\end{array}$ & 395.81 & 396.77 & $\cdots$ & $\cdots$ & $\cdots$ \\
\hline $\begin{array}{l}\text { RPE-CVD }(21 / \mathrm{Jan} / 05) \\
530{ }^{\circ} \mathrm{C} \text { on sapphire }\end{array}$ & 395.88 & 396.94 & $\cdots$ & $\cdots$ & $\cdots$ \\
\hline $\begin{array}{l}\text { RPE-CVD }(24-25 / \mathrm{Jan} / 05) \\
560^{\circ} \mathrm{C} \text { on sapphire }\end{array}$ & 395.80 & 396.84 & $\cdots$ & $\cdots$ & $\cdots$ \\
\hline $\begin{array}{c}\text { rf Sputtered } \\
(4-5 / \mathrm{Jul} / 05) \text { on glass }\end{array}$ & 395.85 & $\cdots$ & 397.23 & 399.12 & 403.05 \\
\hline $\begin{array}{c}\text { rf Sputtered } \\
(6-7 / \mathrm{Jul} / 05) \text { on glass }\end{array}$ & 396.17 & $\cdots$ & 397.71 & 399.61 & 403.88 \\
\hline $\begin{array}{l}\text { Reactive evaporation } \\
(24 / \mathrm{Jan} / 06) \text { on glass }\end{array}$ & 396.07 & $\cdots$ & 397.50 & 399.04 & $\cdots$ \\
\hline $\begin{array}{l}\text { Reactive evaporation } \\
(25 / \mathrm{Jan} / 06) \text { on glass }\end{array}$ & 395.88 & $\cdots$ & 397.38 & $\cdots$ & $\cdots$ \\
\hline $\begin{array}{l}\text { Reactive evaporation } \\
(30 / \mathrm{Jan} / 06) \text { on glass }\end{array}$ & 396.11 & $\cdots$ & 397.48 & 399.29 & $\cdots$ \\
\hline
\end{tabular}

apparent that even if the peak at $396.9 \mathrm{eV}$ for the RPE-CVD and MBE materials is not due to a chemical shift, the binding energy shift represented by this peak is most likely the result of variations in the electronic structure of the $\mathrm{N} 1 s$ core level electrons related to the main InN N $1 s$ peak. In either case it appears that the peak at $\sim 396.9 \mathrm{eV}$ represents a defect species present only in the epitaxial RPE-CVD and MBE grown InN samples. The presence of this defect may be related to the difference in the optical properties observed for the epitaxial and polycrystalline materials studied.

In the case of the first RPE-CVD sample shown in Table II, the low $200{ }^{\circ} \mathrm{C}$ growth temperature for that sample does not allow for the efficient dissociation of the metal organic used as the indium source during the film growth. Consequently, some hydrocarbon is incorporated in the $\mathrm{InN}$ film when using CVD techniques at such low temperatures. This is shown in Ref. 12 where the carbon content of a sister sample was shown to be as high as 10 at. \% in the bulkthough this is still much less than the excess nitrogen that was shown to be present in that same sister sample. If $\mathrm{C}-\mathrm{N}$ bonds are formed during the growth of such a film, then this may contribute to the $\mathrm{N} 1 s$ XPS peak at $399.49 \mathrm{eV}$, shown in Fig. 4(a), as suggested by the work of Kumar et al..$^{31}$ In that same reference, a smaller C-N contribution at $398.1 \mathrm{eV}$ is also identified, and, therefore, there is also the possibility of a small $\mathrm{C}-\mathrm{N}$ contribution in that binding energy region for the $200{ }^{\circ} \mathrm{C}$ RPE-CVD sample examined here. For the rf sputtered and reactively evaporated samples the absence of a carbon source during the actual growth process excludes the presence of a C-N based contribution to the $\sim 399.5 \mathrm{eV}$ peaks seen for those samples.

\section{X-RAY DIFFRACTION ANALYSIS}

In this section we compare XRD data with the ERDA results to provide further insight into nitrogen rich $\mathrm{InN}$ stoichiometry. The use of x-ray diffraction for defect analysis is well established and is based on observations of the 

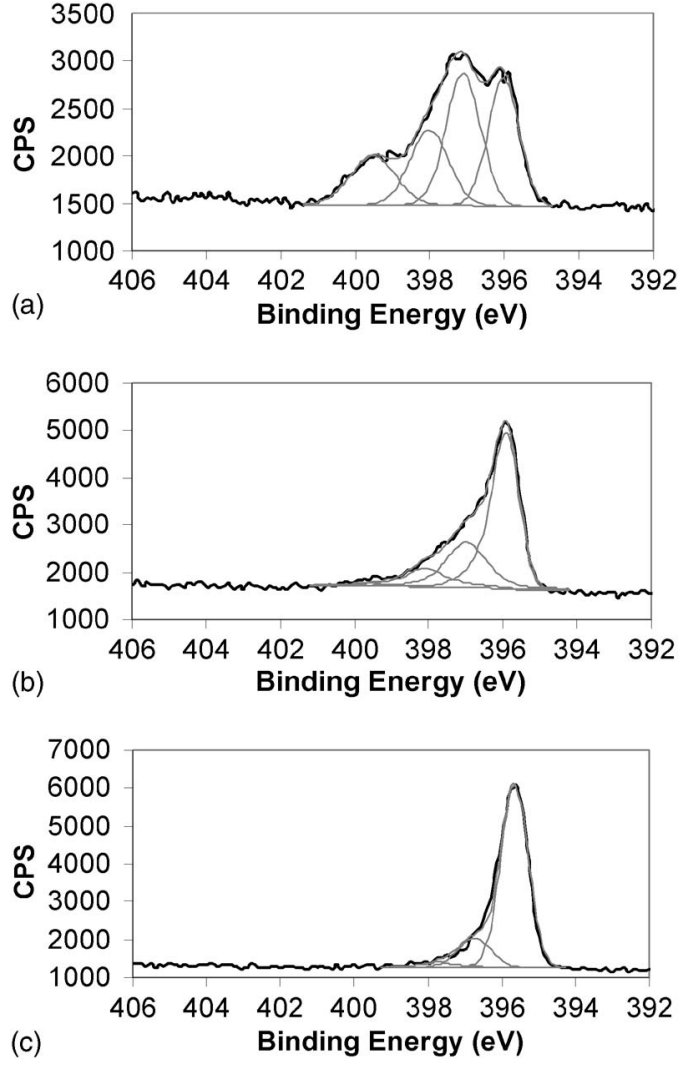

FIG. 4. The figure shows the N $1 s$ spectra obtained by XPS at the surface of indium nitride. The samples were grown by RPE-CVD at (a) $200{ }^{\circ} \mathrm{C}$, (b) $300{ }^{\circ} \mathrm{C}$, and (c) $400{ }^{\circ} \mathrm{C}$. The samples grown at higher temperature have less excess nitrogen and lower carrier concentration (Ref. 13).

changes in the lattice constants and unit cell size. ${ }^{32}$ It is noteworthy that changes in the physical dimension of a film, which may relate to the accumulation of defects at grain boundaries or at other extended defect sites do not generally have a strong influence on the lattice constants measured by
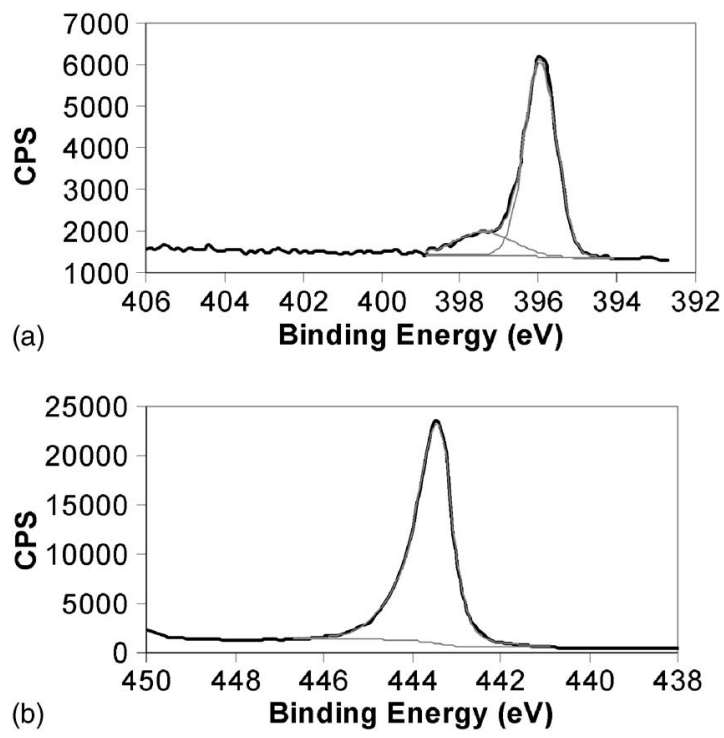

FIG. 5. The figure shows (a) the N $1 s$ spectrum and (b) the In $3 d 5$ spectrum obtained by XPS for indium nitride which had been argon ion etched and heated. The sample was grown on $24-25$ January 2005 at $560{ }^{\circ} \mathrm{C}$ on sapphire by RPE-CVD.
XRD. ${ }^{32}$ The accumulation of indium clusters at grain boundaries, for instance, would have little influence on the data presented here. In essence only defects present in the crystal bulk are observed by this technique. It is quite possible that indium, nitrogen, or even oxygen accumulations may be present at grain boundaries and at other extended defect sites for the material examined. However, this analysis would not identify the presence of those defects. Therefore, unlike many other analysis techniques that cannot distinguish between materials in the crystal bulk and materials at grain boundaries, this study provides specific analyses of the defects present in the crystal bulk.

Used in conjunction with other analysis techniques, XRD based defect analysis can be very effective for nitride films, and there is a long history of its use for such applications. For instance, in 1979, while changing the growth parameters for $\mathrm{GaN}$ to achieve variations in film stoichiometry, Lagerstedt and Monemar ${ }^{33}$ observed a decrease in the lattice constants for a Ga rich material, which they associated with the presence of nitrogen vacancies. They observed a reduction in the lattice parameters following the relationship $\Delta a / a=\Delta c / c=-V_{\mathrm{N}} / N_{\mathrm{GaN}}$, where $V_{\mathrm{N}}$ is the nitrogen vacancy density and $N_{\mathrm{GaN}}$ is the number of GaN molecules. With an increase in growth rate Lagerstedt and Monemar also observed an increase in the $a$ and $c$ lattice parameters, which they attributed to an increase in interstitial species. Overall, up to a $1 \%$ variation in the lattice parameters was observed for undoped $\mathrm{GaN}$.

Kisielowski et al. $^{34}$ were able to use Raman and photoluminescence spectroscopies in conjunction with XRD to demonstrate the coexistence of hydrostatic and biaxial strain in $\mathrm{GaN}$. The biaxial strain resulted from a lattice mismatch, while the hydrostatic strain was shown to result from point defects. Using high resolution XRD, Harutyunyan et al. ${ }^{35}$ built on the earlier work of Kisielowski et al. ${ }^{34}$ analyzing the effect of buffer layer stoichiometry on the strain in $\mathrm{GaN}$ films. Residual hydrostatic strain in the epilayers was attributed to the presence of point defects.

For the case of InN, the XRD stress analysis of Specht et $a l .{ }^{13}$ is particularly useful. In that paper the strain effects of pure hydrostatic, biaxial (basal plane), and uniaxial (linear) stress applied along the $c$ axis have been calculated. The lattice parameters calculated by Specht et al. for pure instances of the three types of stress are shown in Fig. 6.

It is worth reiterating at this point that the MBE and RPE-CVD samples examined here are from the upper and middle sections of the defect line given in the absorption edge versus carrier concentration plot of Fig. 1. That is, they are of medium to high carrier concentration and have been shown to be nitrogen rich. ${ }^{12}$ Samples of lower carrier concentration at the lower end of the defect line, with $\sim 0.65 \mathrm{eV}$ absorption edge, tend to be much thicker than the samples examined here and may exhibit different defect structures and therefore different XRD lattice constants. Certainly in contrast to the material we have examined, there has been some $\sim 0.65 \mathrm{eV}$ material with reported $c$ lattice constant values that are lower than the accepted value, ${ }^{4,36}$ indicating the presence of a different type of defect than those examined here, possibly related to indium rich stoichiometry. 


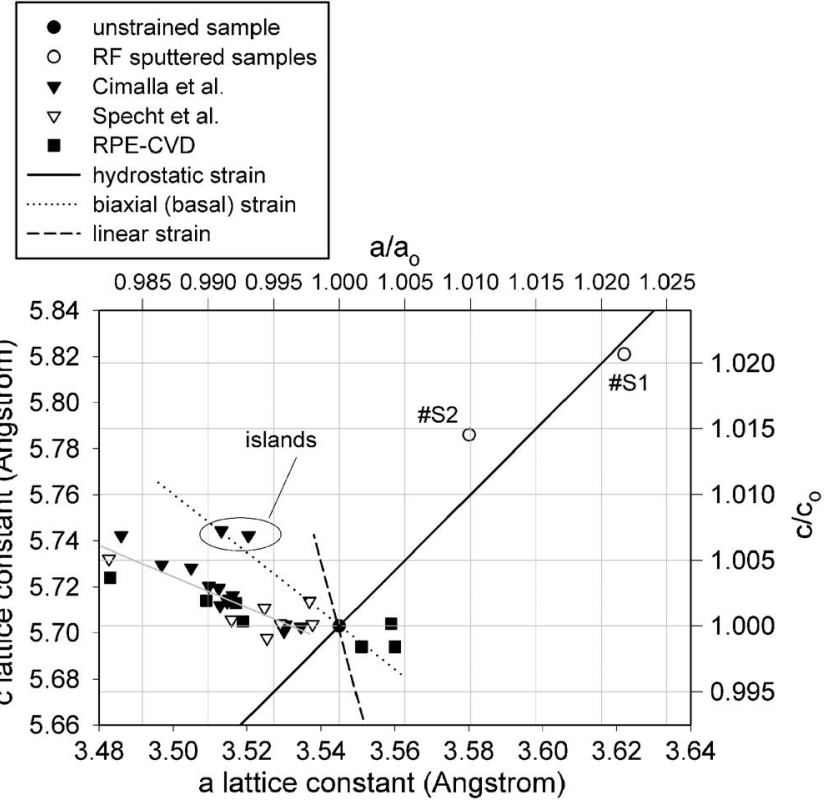

FIG. 6. The figure shows the theoretical strain cases for the three different types of stress shown (i.e., hydrostatic, biaxial, and uniaxial). Data for two nitrogen rich rf sputtered $\mathrm{InN}$ samples (S1 and S2), MBE data reported in the literature and RPE-CVD data, are also shown. An approximate line of fit through the MBE and RPE-CVD data is shown to aid the comparison. The island growth data of Cimalla et al. (Ref. 40) is shown for interest.

rf sputtered InN samples can have quite large amounts of excess nitrogen present. ${ }^{1-3}$ An example of this is shown in Table I. As reported elsewhere, ${ }^{1-3,10,12}$ the table also shows that there is an increase in the $c$-axis lattice constant associated with high levels of excess nitrogen content. These rather large changes in lattice constant indicate that the incorporation of excess nitrogen is accompanied by considerable strain. Some recent reports (for example, Davydov et al. ${ }^{37}$ ) have suggested that this change in lattice constant is the result of $\mathrm{InN}: \mathrm{In}_{2} \mathrm{O}_{3}$ alloying. However, as outlined by Butcher $^{5}$ and by Butcher and Tansley, ${ }^{6}$ this form of alloy does not occur to any significant extent, most oxygen being present in $\mathrm{InN}$ as a segregated amorphous contribution at the InN grain boundaries. This has very recently been confirmed by $\mathrm{Xu}$ et al., who have made observations of oxygen segregation in MBE grown InN that suggest that the solubility of oxygen in $\mathrm{InN}$ is less than $1 \% .^{38}$ The presence of some small portion of the oxygen as a substitutional oxygen on nitrogen donor species is, of course, not excluded, and may possibly be present in some cases. However, the low solubility of oxygen in InN places an upper limit on the presence of this form of oxygen in InN. Hence, the greater portion of the oxygen segregates to grain boundaries. It should be noted, however, that even if the solubility of oxygen in $\mathrm{InN}$ were close to the $1 \%$ upper limit, the hydrostatic lattice strain shown in Fig. 6 is greater than $2 \%$ in both the $c$ and $a$ parameters for sample S1. This corresponds to a lattice volume expansion of over $6 \%$. Therefore, there is no possibility that oxygen could be responsible for the hydrostatic strain shown in the rf sputtered samples, despite the relatively high oxygen content of the rf sputtered films.

The extent and type of stress resulting from excess nitrogen incorporation can be determined by a comparison with the unstressed lattice constants. Here, we use the values of $a=3.545 \AA$ and $c=5.703 \AA$ used by Specht et al. ${ }^{13}$ Both lattice constants are required for a proper evaluation. Goldhahn et $a l .{ }^{36}$ have provided values for two nitrogen rich rf sputtered samples from our laboratory. These samples had $c=5.821$ and $5.786 \AA$ and $a=3.622$ and $3.58 \AA$ for $\operatorname{InN}$ grown on glass $(\mathrm{S} 1)$ and $\mathrm{Si}(\mathrm{S} 2)$, respectively. These values are plotted in Fig. 6. For the case of pure hydrostatic strain, the $c$ and $a$ axes change in proportion to each other, as shown by the straight solid line in the figure. Sample S1 on amorphous glass shows pure hydrostatic stress within the range of experimental error. Sample S2 on Si shows a slight deviation from a pure hydrostatic situation, which may relate to the lattice mismatch between the substrate and the film. However, it appears to still have a very large component of hydrostatic stress apparent. In analogy to $\mathrm{GaN}$, an increase in hydrostatic stress for InN can be related to the homogeneous incorporation of point defects. Hydrostatic strain in InN may result from $\mathrm{N}_{\text {In }}$ and $\mathrm{In}_{\mathrm{N}}$ substitutional point defects, $V_{\mathrm{N}}$ and $V_{\text {In }}$ vacancies and from $\mathrm{N}_{i}$ and $\mathrm{In}_{i}$ interstitial native point defects. Given the large covalent radius of In compared to N, the point defects $\mathrm{In}_{\mathrm{N}}, \mathrm{N}_{i}$, and $\mathrm{In}_{i}$ are expected to result in lattice expansion, whereas the other point defects should lead to lattice compression. Since excess nitrogen has been identified in these films by ERDA measurements, it would appear that the XRD data are commensurate with an increase in some form of interstitial nitrogen or an interstitial complex involving nitrogen. The XPS results in the previous section have shown the presence of a peak at approximately $397.8 \mathrm{eV}$, which may be attributed to an atomic nitrogen species in rf sputtered InN. Therefore, we attribute the positive hydrostatic strain in rf sputtered materials to the presence of a large density of what tentatively appears to be atomic nitrogen interstitials in the lattice.

The N/In ratios shown in Table I for RPE-CVD samples demonstrates that other methods of growth can also access conditions that result in large amounts of excess nitrogen. We have investigated the $a$ and $c$ lattice parameters of such epitaxial films using a high resolution Philips Xpertz X-ray diffraction system and have found that they do not indicate the hydrostatic expansion of the nonepitaxial polycrystalline rf sputtered samples. This is shown in Fig. 6, but is shown elsewhere to also be true for much larger values of film strain that result from exceptionally high values of excess nitrogen in epitaxial InN films. ${ }^{12}$ A comparison of Figs. 1 and 6 in Ref. 12 show the strong correlation between the N/In ratio and the strain in the RPE-CVD based material. This correlation is also shown in our Fig. 7, where the RPE-CVD data of Fig. 6 and other RPE-CVD data are replotted showing the $\mathrm{N} / \mathrm{In}$ ratio for that data versus the $c$ axis lattice parameter. The combined foreign impurity concentrations (hydrogen, oxygen, and carbon) of the samples in Ref. 12 are far too low to account for any notable change in the strain of the samples compared to the much larger amounts of excess nitrogen present. It should be noted that other $\mathrm{InN}$ data, which were shown not to follow the carrier concentration versus absorption edge trend represented by the curve in Fig. 1 (Ref. 39) some of which is represented in Table I-do not follow the trend of Fig. 7. For the readers' interest, Fig. 8 shows the 


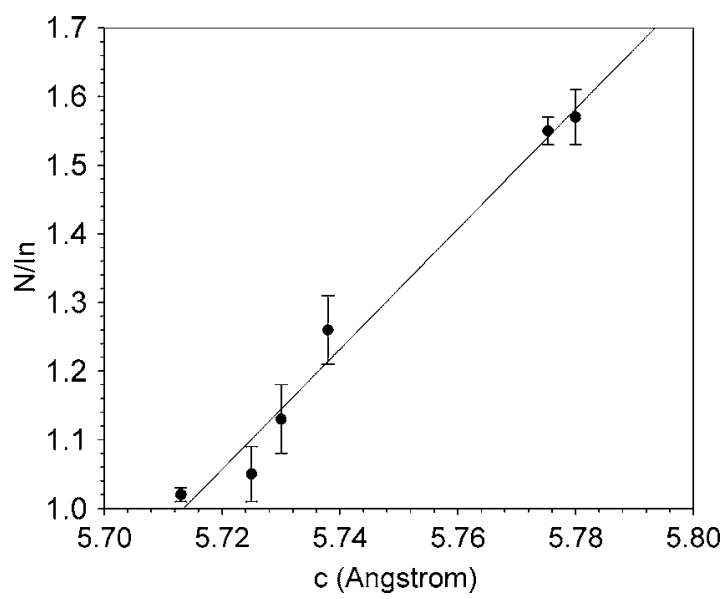

FIG. 7. N/In ratio vs the $c$ axis parameter for RPE-CVD data shown in Fig. 6 and elsewhere. These data follow the carrier concentration vs absorption edge trend of the curve shown in Fig. 1.

correlation between the absorption edge and the $\mathrm{N} / \mathrm{In}$ ratio for RPE-CVD that follows the absorption edge versus carrier concentration trend represented by the curve in Fig. 1.

\section{DISCUSSION}

As shown in Table I and elsewhere, ${ }^{3,21}$ between $2 \%$ and $7 \%$ excess nitrogen has been observed for the thin $(<1 \mu \mathrm{m})$ MBE samples examined here. Similar and higher values have been observed for RPE-CVD grown materials dependent on the growth temperature. ${ }^{12}$ For both MBE (Refs. 7 and 40) and RPE-CVD (Ref. 18) materials the carrier concentration has been observed to decrease as the film thickness is increased, indicating a source of donors near the interface. XRD indicates the origin of a second form of excess nitrogen related to this effect. Figure 6 shows data from Cimalla et al. ${ }^{40}$ and Specht et al. ${ }^{13}$ for MBE grown InN and also some of our data for RPE-CVD samples. The data of Cimalla et al. is for a series of Cornell MBE samples of increasing thickness, the thinnest samples showing the greatest strain, and samples of $1 \mu \mathrm{m}$ thickness showing the least strain on the graph. Cimalla et al. attributed the strain in their samples to a biaxial stress caused by the lattice mismatch

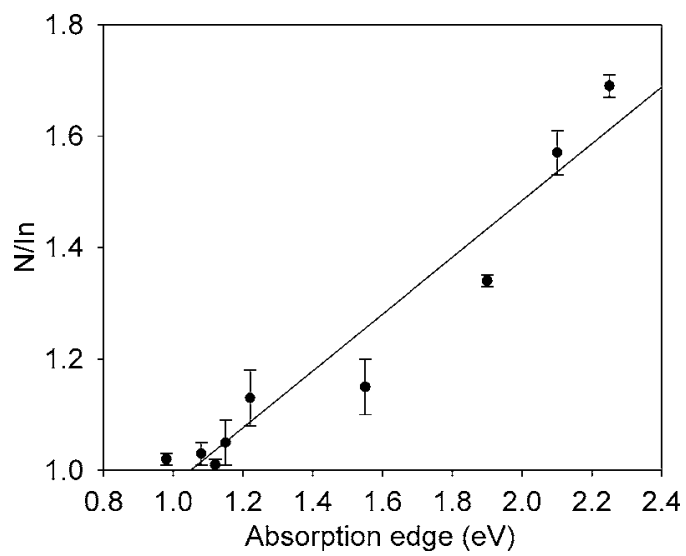

FIG. 8. N/In ratio vs absorption edge data for RPE-CVD data that follow the carrier concentration vs absorption edge trend of the curve shown in Fig. 1 . between the InN and the GaN and AlN buffer layers used. It is evident in the figure, however, that the strain of their samples was not purely biaxial (except perhaps for their reported island growth case, as shown in Fig. 6). Even taking into account the possibility of the unstrained lattice values being closer to those given by other authors, ${ }^{41,42}$ it is apparent that the data of Cimalla et al., ${ }^{40}$ and Specht et al., ${ }^{13}$ and also the RPE-CVD data shown in the figure, all have a reduced lattice volume compared to the case of pure biaxial strain. Vector analysis therefore indicates that a component of either hydrostatic strain or linear strain is present. Hydrostatic strain is the expected result in the case where simple native point defects are incorporated, and given the nonstoichiometry of the material, it is the more likely of the two possibilities. Both of these cases, however, are likely to involve the incorporation of defects, with a higher density of defects being present with higher strain values, i.e., near the substrate interface. Interestingly, the greatest strain observed for the RPE-CVD samples occurred for those samples grown at the lower growth temperatures investigated, i.e., those samples with higher excess nitrogen content ${ }^{12}$ indicating that other growth conditions can be important. It should be noted that in Fig. 6 the more heavily strained RPE-CVD samples, and also the more heavily strained MBE samples studied by Cimalla et al., ${ }^{40}$ were those with the highest carrier concentrations. The introduction of point defects into these heavily strained samples undoubtedly occurs as a means of stress reduction that becomes energetically more favorable as the stress in the film increases. The case for these epitaxial InN samples therefore appears to be analogous to observations of combined hydrostatic and biaxial stress in GaN, where biaxial stress due to lattice mismatch has been shown to be accompanied by hydrostatic stress due to point defects, which themselves result from stoichiometry variations. ${ }^{34,35}$

It is evident in Fig. 6 that a significant reduction of the $c$ lattice parameter occurs for the MBE and RPE-CVD samples compared to the pure biaxial strain case. There are only three of the six simple native defects that could result in this reduction: nitrogen vacancies, indium vacancies, and nitrogenon-indium antisite defects (since the nitrogen ion is smaller than the indium ion). Laakso et al. ${ }^{43}$ carried out positron annihilation experiments on MBE grown samples from the same source as that used by Cimalla et al. and were not able to observe any nitrogen vacancies. Although this does not exclude their presence, the overall nitrogen rich stoichiometry of these thin films $(<1 \mu \mathrm{m})$ would suggest that significant concentrations of nitrogen vacancies are not present. However, indium vacancies were observed by Laakso et al., and their density was found to be considerably higher near the substrate interface. An exact value of the In vacancy concentration at the interface could not be determined by Laakso, though a lower limit of $5 \times 10^{18} \mathrm{~cm}^{-3}$ for the thinnest sample examined $(120 \mathrm{~nm})$ was determined. This value may be as much as an order of magnitude too low to explain the change in the $c$ lattice constant shown in Fig. 6 for samples of Cimalla et al. of $\sim 100 \mathrm{~nm}$ thickness. For samples of that thickness a $0.3 \%-0.5 \%$ difference in the lattice constant is shown, compared to pure biaxial strain (dependent on the exact value of the zero strain lattice constants) and should 
equate to a vacancy concentration estimated at least 5 $\times 10^{19} \mathrm{~cm}^{-3}$. Another problem is that In vacancies are believed to be shallow acceptors in InN rather than donors, ${ }^{17,44}$ so that the presence of In vacancies does not explain the strong $n$-type conductivity of the material, though it may explain the presence of the high levels of compensating acceptors, as have been observed by Look et al. ${ }^{11}$ for Cornell $n$-type MBE materials.

The excess nitrogen observed by ERDA (again for MBE samples from the same source as that examined by Cimalla et al., i.e., Cornell samples) is also not fully explained by the relatively low density of indium vacancies present. The XPS surface results of Table II indicate that at least two forms of nitrogen defect can be present in the epitaxial RPE-CVD and MBE samples. One of these is a relatively low concentration of atomic nitrogen, also observed for the polycrystalline rf sputtered and reactively evaporated materials, that is most likely an interstitial species and possibly a significant donor species for this material, though its presence does not explain the reduction in lattice constants for the MBE and RPE-CVD samples compared to the pure biaxial stress case. The origin of the second, higher density species observed by XPS at a binding energy of $\sim 396.9 \mathrm{eV}$ is less certain. Given the presence of the interstitial species and the presence of indium vacancies, it seems a strong possibility that a mobile interstitial nitrogen atom may be available to fill an indium vacancy. Room temperature mobility of excess nitrogen has been observed in rf sputtered samples. ${ }^{2}$ A high level of nitrogen-on-indium sites would explain both the low $c$ lattice constant observed for the samples of Fig. 6 (compared to the case of pure biaxial strain) and the presence of excess nitrogen in thin MBE samples. The theoretical results of Stampfl et al. $^{17}$ and of Jenkins and Dow ${ }^{44}$ indicate that the nitrogen on the indium antisite defect will act as a deep level trap for both electrons and holes in InN. Hence, if this defect is present, it will be optically active but will not supply donor species. The increased $n$-type carrier concentration observed for thin, more heavily strained MBE samples and for thin, nitrogen rich (also more heavily strained) RPE-CVD samples may be explained by the presence of the interstitial nitrogen species, present in lower concentrations than the $\sim 396.9 \mathrm{eV}$ defect, but as shown in Fig. 4 the $\sim 397.8 \mathrm{eV}$ defect (attributed to interstitial nitrogen) is observed to be present at higher concentrations for those samples with higher carrier concentrations. The donor capability of interstitial nitrogen has not been investigated theoretically for InN. However, its presence in large quantities in some rf sputtered material may explain the high $n$-type carrier concentrations observed for those samples.

One interesting point to note is that the XPS results above confirm the presence of a nitrogen related surface defect for both MBE and RPE-CVD grown InN (the $\sim 396.9 \mathrm{eV}$ defect). Furthermore, this defect does not appear to be present for the $\mathrm{rf}$ sputtered or reactively evaporated nonepitaxial polycrystalline materials. Given that the film surface and the substrate-film interface both represent discontinuities of the film, some defects may be common at both interfaces, though further research may be necessary to confirm this. We suggest, however, that the nitrogen-on- indium antisite defect may be incorporated in the strained region near the substrate interface for InN on GaN or AlN buffer layers, and for InN on sapphire. A higher density of this defect present for thin epitaxial samples and for those grown at lower temperatures, in combination with associated indium vacancies and nitrogen interstitials, as described above, may be responsible for some portion of the upper part of the carrier concentration dependent absorption edge data observed for epitaxial $\mathrm{InN}$ materials and represented by the curve in Fig. 1. In contrast, the presence of large amounts of interstitial atomic nitrogen (without indium vacancies or nitrogen on indium antisite defects) in the polycrystalline materials may dominate their optical absorption properties through different mechanisms (albeit yet to be properly understood) and result in the higher apparent band gap observed for those materials over a wide range of carrier concentrations.

\section{CONCLUSIONS}

We have shown, through a combination of ERDA, XPS, and $\mathrm{XRD}$ results, that at least two distinct nitrogen related defects are observed in indium nitride materials. What appears to be an interstitial form of atomic nitrogen is present in many forms of InN but appears to have a relatively high concentration density in rf sputtered polycrystalline nonepitaxial InN. Another native defect was shown to be prevalent in epitaxial InN grown by MBE or RPE-CVD in strained regions such as that near the interface between the substrate and film, where a combination of a biaxial and what is probably a hydrostatic strain is evident. On the basis of XRD and XPS data we suggest that the source of this defect may be a combination of indium vacancies and a higher density of nitrogen-on-indium antisite defects, though more complex defect structures certainly cannot be ruled out as possible explanations at this stage. The effects of these defects in epitaxial materials were most prominent for thinner films and for films with greater overall excess nitrogen present. This is shown by XRD data, which indicate a stronger deviation from unstrained values for such films. Higher carrier concentrations are also present in such circumstances, though this may be related to the interstitial nitrogen also present at higher concentration. XPS also reveals an increased contribution to a $\mathrm{N} 1 s$ photoelectron peak at $\sim 369.9 \mathrm{eV}$, which may be related to the presence of nitrogen on indium antisite defects in epitaxial materials. We further suggest that the carrier concentration related dependence observed for the optical absorption edge data of MBE and other epitaxial materials is strongly related to the presence of the defects present in this materials. The different defect structures observed for polycrystalline materials, may be related to the different optical absorption edge and electrical properties seen for that material.

\section{ACKNOWLEDGMENTS}

The authors would like to acknowledge the support of an Australian Research Council Discovery grant and of a Macquarie University Research Development grant. They would 
also like to thank Dr. W. Schaff of Cornell and Dr. S. Ivanov of the Ioffe Institute for the supply of samples.

${ }^{1}$ K. S. A. Butcher, M. Wintrebert-Fouquet, Motlan, S. K. Shrestha, H. Timmers, K. E. Prince, and T. L. Tansley, Mater. Res. Soc. Symp. Proc. 743, 707 (2003).

${ }^{2}$ K. S. A. Butcher et al., J. Appl. Phys. 95, 6124 (2004).

${ }^{3}$ H. Timmers, S. K. Shrestha, and A. P. Byrne, J. Cryst. Growth 269, 50 (2004).

${ }^{4}$ T. V. Shubina et al., Phys. Status Solidi A 202, 377 (2005).

${ }^{5}$ K. S. A. Butcher, in InN, an Historic Review: From Obscurity to Controversy, Advanced Material in Electronics, edited by Q. Guo (Research Signpost, Kerala, India, 2004), p. 1.

${ }^{6}$ K. S. A. Butcher and T. L. Tansley, Superlattices Microstruct. 38, 1 (2005).

${ }^{7}$ H. Lu, W. J. Schaff, L. F. Eastman, J. Wu, W. Walukiewicz, D. C. Look, and R. J. Molnar, Mater. Res. Soc. Symp. Proc. 743, 317 (2003).

${ }^{8}$ B. R. Nag, J. Cryst. Growth 269, 35 (2004).

${ }^{9}$ V. M. L. Chin, T. L. Tansley, and T. Osotchan, J. Appl. Phys. 75, 7365 (1994).

${ }^{10}$ K. S. A. Butcher, H. Hirshy, R. M. Perks, M. Wintrebert-Fouquet, and P. P.-T. Chen, Phys. Status Solidi A 203, 66 (2006).

${ }^{11}$ D. C. Look, H. Lu, W. J. Schaff, J. Jasinski, and Z. Liliental-Weber, Appl. Phys. Lett. 80, 258 (2002).

${ }^{12}$ P. P.-T. Chen et al., J. Cryst. Growth 288, 241 (2006).

${ }^{13}$ P. Specht, R. Armitage, J. Ho, E. Gunawan, Q. Yang, X. Xu, C. Kisielowski, and E. R. Weber, J. Cryst. Growth 269, 111 (2004).

${ }^{14}$ J. C. Ho, P. Specht, Q. Yang, X. Xu, D. Hao, and E. R. Weber, J. Appl. Phys. 98, 093712 (2005).

${ }^{15}$ K. S. A. Butcher et al., Phys. Status Solidi C 2, 2263 (2005).

${ }^{16}$ H. Lu, W. J. Schaff, J. Hwang, H. Wu, W. Yeo, A. Pharkya, and L. F. Eastman, Appl. Phys. Lett. 77, 2548 (2000).

${ }^{17}$ C. Stampfl, C. G. Van De Walle, D. Voggel, P. Krugger, and J. Pollman, Phys. Rev. B 61, R7846 (2000).

${ }^{18}$ M. Wintrebert-Fouquet, K. S. A. Butcher, and P. P.-T. Chen, J. Cryst. Growth 269, 134 (2004).

${ }^{19}$ W. A. Grant, in Rutherford Back-Scattering Spectroscopy, Methods of Surface Analysis: Techniques and Applications, edited by J. M. Walls (Cambridge University Press, Cambridge, 1989), p. 299.

${ }^{20} \mathrm{~J}$. M. Walls, in Methods of Surface Analysis, Methods of Surface Analysis: Techniques and Applications, edited by J. M. Walls (Cambridge Univer- sity Press, Cambridge, 1989) p. 1.

${ }^{21}$ S. K. Shrestha, K. S. A. Butcher, M. Wintrebert-Fouquet, and H. Timmers, Nucl. Instrum. Methods Phys. Res. B 219-220, 686 (2004).

${ }^{22}$ S. K. Shrestha and H. Timmers, Nucl. Instrum. Methods Phys. Res. B (in press), available online.

${ }^{23}$ Auger and X-Ray Photoelectron Spectroscopy, Practical Surface Analysis Vol. 1, 2nd ed., edited by D. Briggs and M. P. Seah (Wiley, Chicester, 1990).

${ }^{24}$ T. L. Barr, Modern ESCA: The Principles and Practice of X-ray Photoelectron Spectroscopy (CRC, Boca Raton, FL, 1994).

${ }^{25}$ S. Kumar, L. Mo, Motlan, and T. L. Tansley, Jpn. J. Appl. Phys., Part 135 , 2261 (1996)

${ }^{26}$ J. F. Moulder, W. F. Stickle, P. E. Sobol, and K. D. Bomben, in Handbook of X-Ray Photoelectron Spectroscopy, edited by J. Chastain, p. 22. (Perkin-Elmer, Physical Electronics Division, Eden Prairie, 1992), p. 22.

${ }^{27}$ M. K. Bahl, R. O. Woodall, R. L. Watson, and K. J. Irgolic, J. Chem. Phys. 64, 1210 (1976).

${ }^{28}$ J. A. Schreifels, J. E. Deffeyes, L. D. Neff, and J. M. White, J. Electron Spectrosc. Relat. Phenom. 25, 191 (1982).

${ }^{29}$ M. Wintrebert-Fouquet, K. S. A. Butcher, and Motlan, Phys. Status Solidi C 0, 2785 (2003).

${ }^{30}$ W. F. Egelhoff, Jr., Surf. Sci. Rep. 6, 253 (1987).

${ }^{31}$ S. Kumar, K. S. A. Butcher, and T. L. Tansley, J. Vac. Sci. Technol. A 14, 2687 (1996).

${ }^{32}$ B. Henderson, Defects in Crystalline Solids (Arnold, London, 1972), pp. 36-37, 136-138.

${ }^{33}$ O. Lagerstedt and B. Monemar, Phys. Rev. B 19, 3064 (1979).

${ }^{34}$ C. Kisielowski et al., Phys. Rev. B 54, 17745 (1996).

${ }^{35}$ V. S. Harutyunyan, A. P. Aivazyan, E. R. Weber, Y. Kim, Y. Park, and S. G. Subramanya, J. Phys. D 34, A35 (2001).

${ }^{36}$ R. Goldhahn et al., Mater. Res. Soc. Symp. Proc. 743, 361 (2003).

${ }^{37}$ V. Yu. Davydov et al., Phys. Status Solidi B 234, 787 (2002).

${ }^{38}$ X. Xu, P. Specht, R. Armitage, J. C. Ho, E. R. Weber, and C. Kisielowski, Appl. Phys. Lett. 87, 092102 (2005).

${ }^{39}$ K. S. A. Butcher et al., Phys. Status Solidi C 2, 2263 (2005).

${ }^{40}$ V. Cimalla et al., Phys. Status Solidi C 0, 2818 (2003).

${ }^{41}$ W. Paszkowicz, R. Cerny, and S. Krukowski, Powder Diffr. 18, 114 (2003).

${ }^{42}$ V. Yu. Davydov et al., Phys. Status Solidi B 229, R1 (2002).

${ }^{43}$ A. Laakso et al., J. Cryst. Growth 269, 41 (2004).

${ }^{44}$ D. W. Jenkins and J. D. Dow, Phys. Rev. B 39, 3317 (1989). 